\title{
Improved real-time recordings using the fourth generation cryoballoon technology - detection of dual fascicle electrograms
}

\author{
Vedran Velagić ${ }^{1}$, Giacomo Mugnai ${ }^{2}$, Vedran Pasara ${ }^{1}$, Ivan Prepolec ${ }^{1}$, Mislav Puljevic ${ }^{1}$, \\ Borka Pezo-Nikolic ${ }^{1}$, Davor Puljevic ${ }^{1}$, Jure Samardzic ${ }^{1}$, Maja Cikes ${ }^{1}$, and Davor Milicic ${ }^{1}$ \\ ${ }^{1}$ University of Zagreb School of Medicine, University Hospital Centre Zagreb, Zagreb, \\ Croatia \\ ${ }^{2}$ Division of Cardiology, West Vicenza General Hospitals, Vicenza, Italy
}

April 28, 2020

\begin{abstract}
Introduction: We aimed to analyze the rate of visualization of real-time (RT) recordings and dual fascicle electrograms in our first series of patients treated with the fourth generation cryoballoon (CB) device. Methods: All consecutive patients who underwent $\mathrm{CB}$ ablation using the fourth generation technology were included in the analysis. In all procedures we used a $28 \mathrm{~mm}$ CB placed via a single transseptal puncture guided by intracardiac ultrasound. A $20 \mathrm{~mm}$ octapolar intraluminal circular catheter was used for intracranial recordings. A single 180 seconds freeze strategy was employed. Results: A total of 123 patients (72.9\% male, mean age $60.1 \pm 10.9$ years) were enrolled in the study. RT recordings were detected in 445 (86.2\%) pulmonary veins (PVs). Specifically, RT recordings were visualized in 115 left superior PVs (89.2\%), 107 left inferior PVs (82.9\%), 118 right superior PVs (91.4\%) and 105 right inferior PVs (81.3\%). Furthermore, in 23 of 516 PVs (4.4\%), two fascicle electrograms were detected. Dual fascicles were most commonly observed in left superior PV (6.2\%). In both inferior PVs dual fascicles were observed in $4.6 \%$ while this phenomenon was least frequent in right superior PV $(2.3 \%)$. Conclusion: By using the fourth-generation CB we report a specific pattern of isolation represented by a sequential isolation of two apparently distinct PV fascicles during a single CB freeze delivery. This phenomenon occurred in $4.4 \%$ of PV ablations. Of note, the rate of visualization of RT isolation with this novel CB was very high and could be documented in $86.2 \%$ of PVs.
\end{abstract}

\section{Hosted file}

fascikli pejper rev 5.doc available at https://authorea.com/users/310036/articles/440870-improvedreal-time-recordings-using-the-fourth-generation-cryoballoon-technology-detection-of-dualfascicle-electrograms 